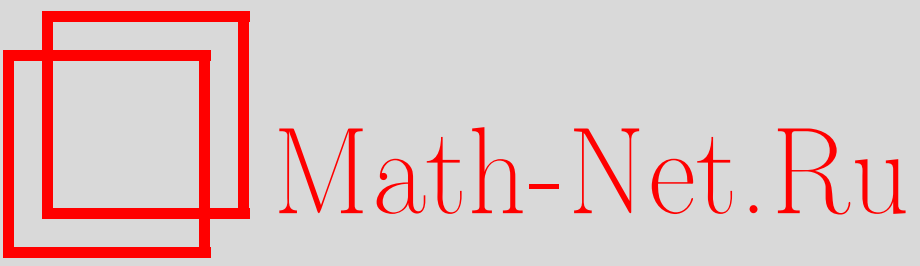

Ю. А. Кашлев, Неравновесная статистическая термодинамика атомов позитрония, движущихся в твердых телах, ТМФ, 2005, том 142, номер 1, 112-126

DOI: https://doi.org/10.4213/tmf1763

Использование Общероссийского математического портала Math-Net.Ru подразумевает, что вы прочитали и согласны с пользовательским соглашением

http://www . mathnet.ru/rus/agreement

Параметры загрузки:

IP : 54.92 .164 .108

26 апреля 2023 г., 12:40:39 


\section{НЕРАВНОВЕСНАЯ СТАТИСТИЧЕСКАЯ ТЕРМОДИНАМИКА АТОМОВ ПОЗИТРОНИЯ, ДВИЖУЩИХСЯ В ТВЕРДЫХ ТЕЛАХ}

В рамках методов неравновесной статистической термодинамики вычислена квазитемпература атомов позитрония, движущихся в твердом теле. Квазитемпература представлена в виде перенормированной температуры термостата. Перенормировка обусловлена учетом скорости образования позитрония, кинетическими эффектами взаимодействия и динамическими эффектами столкновений. Получено выражение для времени термализации атомов позитрония.

Ключевые слова: неравновесная статистическая термодинамика, атом позитрония, твердое тело, термодинамические параметры, квазитемпература, коэффициент переноса, матрица релаксации, термализация.

\section{1. ВВОДНЫЕ ЗАМЕЧАНИЯ}

Статья посвящена исследованию термодинамики атомов позитрония (АП), движущихся в кристаллах. АП образуются в твердых телах при электрон-позитронных столкновениях. Вероятность рождения АП в элементарном акте рассеяния достаточно высока; например, в диэлектрике она оценивается как $2 / 3$ [1]. При рассмотрении процесса образования АП в большинстве случаев используется энергетическая диаграмма, согласно которой АП рождаются в так называемой зоне образования шириной $\sim 7$ эВ [1]. Верхняя граница зоны находится вблизи края внутренней ионизации кристалла. Что же касается нижней границы, то она соответствует энергиям, при которых скорости АП достигают $v(\bar{\varepsilon}) \sim 10^{8} \mathrm{~cm} / \mathrm{c}$. Причем энергия образования АП в диэлектриках всегда положительна, тогда как в металлах ситуация сложнее.

Запишем энергию образования в виде [2] $\phi_{\mathrm{ps}}=\phi_{\mathrm{p}}+\phi_{e}-E_{\mathrm{b}}$. Здесь $E_{\mathrm{b}}=R y / 2-$ энергия связи АП в основном состоянии. Энергия $E_{\mathrm{b}}$ отдается системе в момент образования АП, поэтому она входит в $\phi_{\mathrm{ps}}$ с отрицательным знаком. Функция $\phi_{\mathrm{p}}$ (work function [2]) связана с химическим потенциалом позитронов простым соотношением $\phi_{\mathrm{p}}=-\mu_{\mathrm{p}}$. В свою очередь химический потенциал определяется как $\mu_{\mathrm{p}}=-E_{\mathrm{corr}}-E_{0}$; в соответствии

${ }^{*}$ Институт металлургии и материаловедения им. А. А. Байкова РАН, Москва, Россия. Е-mail: pm@ultra.imet.ac.ru 
с принятыми в работе [2] обозначениями $E_{\text {corr }}$ - корреляционная энергия позитрона в электронном газе, $E_{0}$ - добавка к энергии позитрона, обусловленная позитрон-ионным взаимодействием. В качестве функции $\phi_{e}$ для электрона, как правило, принимается химический потенциал (с отрицательным знаком) электронов при нулевой температуpe: $\phi_{e}=-\mu_{e}$. Расчет величин $E_{\text {corr }}, E_{0}$ и $\mu_{e}$ показал, что определенная на их основе энергия $\phi_{\mathrm{ps}}$ положительна только в случае щелочных металлов с низкой плотностью электронов $\left(4<r_{\mathrm{S}}<5.6\right)[2]$, [3]. Поэтому в дальнейшем речь пойдет только об АП в диэлектриках и щелочных металлах.

В настоящей работе на основе методов неравновесной статистической термодинамики [4] исследовано стационарное квазиравновесное состояние АП на дне зоны образования. Вычислены коэффициенты переноса и квазитемпература подсистемы АП в состоянии квазиравновесия, построено уравнение баланса энергии. Вместе с тем рассмотрена стадия термализации, в период которой скорости АП снижаются до тепловых.

\section{2. ЭНТРОПИЯ, ТЕРМОДИНАМИЧЕСКИЕ ПАРАМЕТРЫ ПОДСИСТЕМЫ АП, УРАВНЕНИЕ БАЛАНСА ЭНЕРГИИ АП}

Считая АП самостоятельной термодинамической подсистемой, разделим полную систему на две слабо взаимодействуюшие подсистемы: индекс $i=1$ соответствует термостату (решетка плюс электронный газ), индекс $i=2$ - газу “горячих" атомов (АП). В рамках сокрашенного описания состояние системы характеризуется совокупностью средних значений двух переменных $P_{m i}$, где $m=1,2$, а именно, $P_{1 i}=H_{i}$-гамильтониан и $P_{2 i}=N_{i}$ - число частиц $i$-й подсистемы. Полный гамильтониан АП представим в виде $H_{2}=H_{0}^{(2)}+H_{\mathrm{int}}^{(2)}$, где $H_{0}^{(2)}$ - гамильтониан идеального газа $\mathrm{A \Pi ,} H_{\mathrm{int}}^{(2)}$ - гамильтониан взаимодействия АП с термостатом. Взаимодействие между АП не рассматривается, поскольку плотность числа частиц в подсистеме $\{2\}$ считается достаточно малой.

В соответствии с монографией [4] функционал энтропии можно записать в виде

$$
S(t, 0)=\phi(t)+\sum_{m i} P_{m i} F_{i m}(t)
$$

где

$$
\phi(t)=\ln \operatorname{Sp} \exp \left\{-\sum_{m i} P_{m i} F_{i m}(t)\right\}, \quad F_{i m}(t)=\frac{\delta S(t)}{\delta\left\langle P_{m i}\right\rangle^{t}}
$$

Представим неравновесный статистический функционал в виде разложения по степеням потенциала взаимодействия. В системе единиц, в которой $\hbar=k_{\mathrm{B}}=1$, с точностью до членов первого порядка имеем

$$
\begin{aligned}
\rho(t)= & \rho_{l}(t)+\int_{-\infty}^{0} d t^{\prime} e^{\epsilon t^{\prime}} \int_{0}^{1} d \tau e^{-\tau S_{0}(t, 0)} \Delta\left\{\left[F_{21}\left(t+t^{\prime}\right)-\beta_{1}\right] \dot{H}_{2}\left(t^{\prime}\right)\right\} \times \\
& \times e^{(\tau-1) S_{0}(t, 0)}-\int_{0}^{1} d \tau e^{-\tau S_{0}(t, 0)}\left\{\beta_{1} H_{\mathrm{int}}^{(2)}\right\} e^{(\tau-1) S_{0}(t, 0)} .
\end{aligned}
$$


Здесь $\rho_{l}(t)=e^{-S(t, 0)}$ - квазиравновесное распределение, соответствующее экстремуму информационной энтропии [4],

$$
\begin{gathered}
S_{0}(t, 0)=\phi_{0}(t)+F_{21}(t)\left\{H_{2}-\tilde{\mu}_{2} N_{2}\right\}+\widetilde{B}, \\
\phi_{0}(t)=\ln \operatorname{Sp} \exp \left\{-F_{21}(t)\left(H_{2}-\tilde{\mu}_{2} N_{2}\right)-\widetilde{B}\right\}, \\
\widetilde{B}=\beta_{1}\left(H_{1}-\mu_{1} N_{1}\right), \quad \tilde{\mu}_{2}=\mu_{2}-\varepsilon, \quad \Delta A=A-\langle A\rangle^{t}, \quad \epsilon \rightarrow+0 .
\end{gathered}
$$

Параметры $F_{i m}(t)$, вошедшие в формулы (1), подбираются таким образом, чтобы истинные средние набора величин $\left\langle P_{m i}\right\rangle^{t}=\operatorname{Sp}\left\{\rho(t) P_{m i}\right\}$ были равны их квазиравновесным средним $\left\langle P_{m i}\right\rangle_{l}^{t}=\operatorname{Sp}\left\{\rho_{l}(t) P_{m i}\right\}$. Тогда квазиравновесное распределение становится максимально близким к истинному неравновесному распределению. При таком выборе $F_{i m}(t)$ являются термодинамическими параметрами: $1 / F_{11}=1 / \beta_{1}=T_{1}$ - температура термостата, $1 / F_{21}(t)$ - квазитемпература подсистемы $\{2\}, F_{12}=-\beta_{1} \mu_{1}, \mu_{1}$ химический потенциал термостата, $F_{22}(t)=-F_{21}(t) \mu_{2}, \mu_{2}=\mu_{\mathrm{p}}+\mu_{e}$ - химический потенциал подсистемы $\{2\}, \lambda=e^{-F_{22}(t)}$ - абсолютная активность АП; $\varepsilon=\left(1 / \bar{N}_{2}\right)\left\langle H_{2}^{0}\right\rangle_{0}$, где усреднение выполняется с помошью статистического оператора $\rho_{0}=e^{-S_{0}(0,0)}, \bar{N}_{2}$ число АП. Используя выражение для функционала Масье-Планка $\phi_{\mathrm{p}}$, нетрудно вывести соотношения, определяющие флуктуации величин $P_{m i}$ :

$$
\frac{\delta^{2} \phi(t)}{\delta F_{i m}(t) \delta F_{j n}(t)}=-\frac{\delta\left\langle P_{n j}\right\rangle^{t}}{\delta F_{i m}(t)}=-\frac{\delta\left\langle P_{m i}\right\rangle^{t}}{\delta F_{j n}(t)}=\left(P_{n j}, P_{m i}\right)^{t},
$$

где

$$
\left(P_{n j}, P_{m i}\right)^{t}=\int_{0}^{1} d \tau \operatorname{Sp}\left\{P_{n j} e^{-\tau S_{0}(t, 0)} \Delta P_{m i} e^{(\tau-1) S_{0}(t, 0)}\right\} .
$$

Используя термодинамические соотношения (2), установим связь квазитемпературы подсистемы $\{2\}$ с фллукуацией энергии при условии конечности температуры термостата. Получаем

$$
\frac{1}{F_{21}(t)}=\left\{\frac{1}{C_{V}}\left(H_{2}, H_{2}\right)^{t}\right\}^{1 / 2},
$$

где $C_{V}$ - удельная теплоемкость газа АП. В стационарном квазиравновесном состоянии соотношение (3a) сушественно упрошается. В этом случае (steady state) с использованием (2б), (3а) находим

$$
\left(\frac{1}{F_{21}}\right)_{\mathrm{ss}} \equiv \frac{1}{\beta_{2}}=\left\{\frac{1}{C_{V}}\left(H_{2}, H_{2}\right)\right\}^{1 / 2},
$$

где

$$
\left(H_{2}, H_{2}\right)=\int_{0}^{1} d \tau \operatorname{Sp}\left\{H_{2} e^{-\tau S_{0}(0,0)} \Delta H_{2} e^{(\tau-1) S_{0}(0,0)}\right\} .
$$

Для построения уравнения баланса энергии АП в квазиравновесном состоянии (или вблизи него) необходимо вычислить, с одной стороны, мощность энергии, вводимой в подсистему $\{2\}$ за счет внешнего излучения, с другой - мошность энергии, переданной 
термостату. Средняя скорость увеличения плотности внутренней энергии $\mathrm{AП} E_{2}(t)=$ $\left\langle H_{2}\right\rangle^{t}$ в случае облучения диэлектрика равна

$$
\frac{1}{\bar{N}_{2}}\left\langle H_{2}\right\rangle^{t}\left(\tilde{N} \tilde{\phi} \sigma_{\mathrm{f}}\right)
$$

Здесь $\tilde{\phi}$ - плотность потока бомбардируюших позитронов, $\sigma_{\mathrm{f}}$ - сечение столкновений позитронов с электронами (из числа электронов атомных остовов), $\widetilde{N}$ - плотность атомов решетки, $\widetilde{N} \tilde{\phi} \sigma_{\mathrm{f}}$ - скорость образования АП в единице объема. Объем системы для простоты принят равным единице. Отметим, что при переходе от выражения, записанного для АП в диэлектрике, к выражению для АП в металле $\widetilde{N}$ следует заменить на плотность электронов проводимости.

В стационарном квазиравновесном состоянии скорость увеличения внутренней энергии можно представить в развернутом виде, если воспользоваться выражением [5]

$$
\begin{gathered}
\frac{1}{\bar{N}_{2}}\left\langle H_{2}\right\rangle=\bar{\varepsilon} f_{1}\left(F_{21}\right), \\
f_{1}\left(F_{21}\right)=2\left[\varphi\left(F_{21}\right)\right]^{-2}\left\{1-\left[1+\varphi\left(F_{21}\right)\right] e^{-\varphi\left(F_{21}\right)}\right\},
\end{gathered}
$$

которое применимо для подсистемы частиц, движущихся хаотически, а значит, и для АП на дне зоны. В формуле (4) $\varphi\left(F_{21}\right)=\pi F_{21} \bar{\varepsilon} / 2$, а средняя энергия $\bar{\varepsilon}$, как и в статистической теории [6], [7], записывается в пределе $1 / F_{21} \rightarrow \infty$. Теперь для подсистемы $\{2\}$ уравнение баланса энергии можно записать, используя выражение (4). Получаем

$$
\left\langle\frac{d E_{2}}{d t}\right\rangle=\bar{\varepsilon} f_{1}\left(F_{21}\right) \tilde{N} \tilde{\phi} \sigma_{f}-L_{\dot{H}_{2} \dot{H}_{2}} X
$$

Здесь $L_{\dot{H}_{2} \dot{H}_{2}}$ - коэффициент переноса, вошедший в линейное соотношение [4] между потоком энергии и термодинамической силой $X=\beta_{1}-F_{21}$. Второй член в правой части (5) - энергия, которую в единицу времени теряет подсистема $\{2\}$ в результате двух диссипативных процессов: фононного рассеяния АП и возбуждения атомами позитрония электрон-дырочных пар.

\section{3. КОЭФФИЦИЕНТЫ ПЕРЕНОСА}

3.1. Фононное рассеяние АП в диэлектрике. Будем рассматривать внутризонное рассеяние АП $(1 S, K) \rightarrow(1 S, K+q)$ на тепловых колебаниях атомов решетки. В этом случае оптическая модель колебаний дает малый вклад в кинетические коэффициенты [8], поэтому ограничимся учетом взаимодействия АП с акустическими фононами. В области малых передач импульса $q$ коэффициент переноса, вошедший в уравнение баланса (5), может быть записан в форме [5]

$$
L_{\dot{H}_{2} \dot{H}_{2}}^{(\mathrm{ph})}=\frac{1}{2} \int d \omega \omega^{2} \frac{1}{2 \pi^{2}} \int d \mathbf{q}\left|V_{1}(q)\right|^{2} \beta_{1} \omega S^{(\mathrm{ph})}(\mathbf{q}, \omega) \delta((\mathbf{q v}(\bar{\varepsilon}))-\omega) .
$$


Здесь использованы обозначения: $V_{1}(q)$ - фурье-компонента деформационного потенциала взаимодействия АП с решеткой [8], [9]:

$$
\left|V_{1}(q)\right|^{2}=g^{2} \frac{q}{2 M \widetilde{N} v_{\mathrm{S}}}
$$

$g$ - константа связи, которая в теории миграции [9] по порядку величины считается равной энергии связи комплекса позитрон-вакансия; $M$ и $v_{\mathrm{S}}$ - масса атома решетки и скорость звука, соответственно;

$$
S^{(\mathrm{ph})}(\mathbf{q}, \omega)=\frac{1}{2 \pi} \int_{0}^{\infty} d t e^{i \omega t}\left\langle\rho_{-\mathbf{q}}^{(i)} \rho_{\mathbf{q}}^{(i)}(t)\right\rangle_{0}
$$

- динамический формфактор,

$$
\left\langle\rho_{-\mathbf{q}}^{(i)} \rho_{\mathbf{q}}^{(i)}(t)\right\rangle_{0}=e^{-2 \widetilde{W}} \sum_{\mathbf{R}} e^{-i \mathbf{q} \mathbf{R}} \exp \langle(\mathbf{q u}(0, t))(\mathbf{q u}(\mathbf{R}, t))\rangle_{0},
$$

$\rho_{\mathbf{q}}^{(i)}$ - фурье-компонента плотности атомов решетки, $\mathbf{u}(\mathbf{R}, t)$ - смешение атома из положения равновесия $\mathbf{R}, e^{-2 \widetilde{W}}-$ фактор Дебая-Валлера. Проинтегрировав (6) по угловым переменным вектора q, имеем

$$
L_{\dot{H}_{2} \dot{H}_{2}}^{(\mathrm{ph})}=\frac{1}{2 \pi^{2}} v(\bar{\varepsilon}) \beta_{1} \int d \omega \omega^{2} \int d q q^{3}\left|V_{1}(q)\right|^{2} S^{(\mathrm{ph})}(q, \omega)\left|\int_{0}^{\infty} d t^{\prime} e^{-i \omega t^{\prime}} j_{1}\left(q v(\bar{\varepsilon}) t^{\prime}\right)\right|,
$$

где $j_{1}(x)$ - цилиндрическая функция Бесселя.

Теперь выполним в выражении (7) интегрирование по $d t^{\prime}$ и $d \omega$, предварительно разложив динамический формфактор в ряд по степеням смещения. Если ограничиться рассмотрением однофононных и двухфононных процессов рассеяния АП, то в формуле (7) достаточно сохранить члены порядка $u$ и $u^{2}$. Тогда коэффициент переноса (7) запишется в виде

$$
\begin{gathered}
L_{\dot{H}_{2} \dot{H}_{2}}^{(\mathrm{ph})}=L_{\dot{H}_{2} \dot{H}_{2}}^{(1)}+L_{\dot{H}_{2} \dot{H}_{2}}^{(2)}, \\
L_{\dot{H}_{2} \dot{H}_{2}}^{(1)}=\bar{\varepsilon} T_{1} \frac{1}{\tau_{1}}, \quad L_{\dot{H}_{2} \dot{H}_{2}}^{(2)}=\bar{\varepsilon} T_{1} \frac{1}{\tau_{2}},
\end{gathered}
$$

причем в модели Дебая времена релаксации $\tau_{1}$ и $\tau_{2}$, соответствуюшие однофононному и двухфононному рассеянию, равны

$$
\begin{gathered}
\frac{1}{\tau_{1}}=\frac{1}{3 \pi^{2}} \frac{1}{M \widetilde{N}} \int_{0}^{q_{\mathrm{D}}} d q q^{6} \frac{1}{2 M \omega_{q}}\left|V_{1}(q)\right|^{2} \beta_{1}^{2} \nu\left(\omega_{q}\right) \\
\frac{1}{\tau_{2}}=\frac{4}{3 \pi^{2}} \frac{1}{M \widetilde{N}} \int_{0}^{q_{\mathrm{D}}} d q q^{6}\left[\frac{1}{\omega_{q}^{3}}\left|V_{1}(q)\right|^{2}\right]^{2} \frac{1}{M} \int_{0}^{\Delta_{q}} d \widetilde{\omega} \widetilde{\omega}\left(\Delta_{q}-\widetilde{\omega}\right) \beta_{1}^{2} \nu(\widetilde{\omega}) \nu\left(\Delta_{q}-\widetilde{\omega}\right) \\
\nu(\omega)=\left[e^{\beta_{1} \omega}-1\right]^{-1}
\end{gathered}
$$

Здесь $q_{\mathrm{D}}$ - дебаевский импульс, $\Delta_{q}$ - энергия, переданная АП при поглошении (испускании) двух фононов. 
3.2. Электронное рассеяние АП в металлах. Рассмотрим рассеяние АП с энергией, соответствующей дну зоны образования $(\sim 3 \div 5$ эВ), на электронах проводимости. Учтем, что в шелочных металлах энергия плазмонов значительно больше указанных энергий АП [10]. В этих условиях передача энергии из подсистемы $\{2\}$ в термостат путем рождения плазмонов невозможна (то же утверждается в работе [11]). Рассматриваемая нами физическая ситуация совершенно иная, чем при прохождении высокоэнергетических позитронов ( 1 кэВ), когда диссипация энергии обусловлена плазмонами. Будем считать, что отвод энергии полностью определяется процессами возбуждения электрон-дырочных пар вблизи поверхности Ферми (в дальнейшем $\varepsilon_{\mathrm{F}}-$ энергия Ферми).

АП можно рассматривать как водородоподобный атом [1], [8]. Эта упрошенная модель принимается в нашей работе. Так что будем использовать в качестве потенциала взаимодействия АП с электронами потенциал, усредненный по основному состоянию собственного электрона $\psi_{0}$ :

$$
V_{2}(q)=\frac{4 \pi e^{2}}{q^{2}}\left\{1-\int d r \frac{4 \pi}{q} r \sin (q r)\left|\psi_{0}(r)\right|^{2}\right\}
$$

где $\left|\psi_{0}(r)\right|^{2}=\pi^{-1} a_{\mathrm{B}}^{-3} e^{-2 r / a_{\mathrm{B}}}, a_{\mathrm{B}}-$ боровский радиус.

Общий вид коэффициента переноса в условиях упругого внутризонного рассеяния с мальми передачами импульса известен [12]:

$$
L_{\dot{H}_{2} \dot{H}_{2}}^{(e)}=\frac{1}{2} \int_{-\infty}^{\infty} d \omega \omega^{2} \int d \mathbf{q} \mathcal{P}(\mathbf{q}, \omega)
$$

где

$$
\mathcal{P}(\mathbf{q}, \omega) d \mathbf{q} d \omega=-\frac{1}{(2 \pi)^{2}}\left|V_{2}(q)\right|^{2} S^{(e)}(\mathbf{q}, \omega) \delta((\mathbf{q v}(\bar{\varepsilon}))-\omega) d \mathbf{q} d \omega
$$

- вероятность перехода, а динамический формфактор выражается через фурье-компоненту (по временно́й переменной) функции Грина [4]

$$
\begin{gathered}
G(\mathbf{q}, t) \equiv\left\langle\left\langle\left\langle\rho_{\mathbf{q}}(t), \stackrel{+}{\rho}_{\mathbf{q}}\right\rangle\right\rangle=-i \theta(t)\left\langle\left[\rho_{\mathbf{q}}(t), \stackrel{+}{\rho}_{\mathbf{q}}(0)\right]\right\rangle_{0}\right. \\
S^{(e)}(\mathbf{q}, \omega)=\frac{1}{\pi} \operatorname{Im}\left\langle\left\langle\rho_{\mathbf{q}} \mid \stackrel{+}{\rho}_{\mathbf{q}}\right\rangle\right\rangle_{\omega}
\end{gathered}
$$

$\rho_{\mathbf{q}}-$ фурье-компонента флуктуации электронной плотности.

Преобразуем выражение (10а). Во-первых, ограничиваясь рассмотрением электронного газа при нулевой температуре, вынесем из-под знака интеграла по $d \omega$ множитель $\omega$ в точке $\omega=(3 / 5) \varepsilon_{\mathrm{F}}$, соответствующий средней энергии электрона в основном состоянии (указанная аппроксимация обеспечивает удовлетворительную точность [12]). Во-вторых, выполним в (10a) интегрирование по угловым переменным q и воспользуемся аппроксимацией $j_{1}(q v t) \simeq q v t / 3$, которая применима в области малых скоростей. В 
заключение в полученном таким образом выражении выполним интегрирование по $d \omega$, используя теорему вычетов. В результате получаем

$$
L_{\dot{H}_{2} \dot{H}_{2}}^{(e)}=-\left.\frac{1}{4 \pi^{4}} \varepsilon_{\mathrm{F}} v^{2}(\bar{\varepsilon}) \int d q q^{4}\left|V_{2}(q)\right|^{2}\left\{\frac{d}{d \omega} \operatorname{Im} G(q, \omega)\right\}\right|_{\omega=0} .
$$

Вычисление производной функции Грина, выполненное в приближении Хартри [13], приводит к результату

$$
\left.\frac{d}{d \omega} \operatorname{Im} G(q, \omega)\right|_{\omega=0}=\left\{\begin{array}{lll}
-\left(2 q v_{\mathrm{F}}\right)^{-1} \mathcal{N}\left(\varepsilon_{\mathrm{F}}\right)\left[1+\frac{k_{\mathrm{TF}}^{2}}{q^{2}} f_{2}(q)\right]^{-2} & \text { при } & 0<q<2 k_{\mathrm{F}} \\
0 & \text { при } \quad q>2 k_{\mathrm{F}} .
\end{array}\right.
$$

Здесь $k_{\mathrm{F}}=m_{e} v_{\mathrm{F}}, v_{\mathrm{F}}-$ скорость $Ф е р м и, m_{e}-$ масса электрона, $k_{\mathrm{TF}}-$ обратный радиус экранирования Томаса-Ферми, $\mathcal{N}\left(\varepsilon_{\mathrm{F}}\right)$ - плотность электронных состояний на единицу энергии вблизи поверхности Ферми,

$$
f_{2}(q)=1-q^{2}\left[2\left(q^{2}+k_{\mathrm{F}}^{2}\right)\right]^{-1} .
$$

Теперь введем в выражениях (11) и (12) новую переменную $\xi=\left(q /\left(2 k_{\mathrm{F}}\right)\right)^{2}$. Имеем

$$
L_{\dot{H}_{2} \dot{H}_{2}}^{(e)}=\bar{\varepsilon} \varepsilon_{\mathrm{F}} \frac{1}{\tau_{3}}
$$

где

$$
\frac{1}{\tau_{3}}=\frac{2}{\pi^{4}} \varepsilon_{\mathrm{F}} \int_{\xi_{\min }}^{1} d \xi \xi^{3} \frac{1}{(\xi+\gamma)\left(\xi+\alpha f_{2}(\xi)\right)^{2}} .
$$

Здесь использованы обозначения: $\alpha=e^{2} /\left(\pi v_{\mathrm{F}}\right)=\left(k_{\mathrm{TF}} /\left(2 k_{\mathrm{F}}\right)\right)^{2}, \gamma=\left(2 k_{\mathrm{F}} a_{\mathrm{B}}\right)^{-2}$. Интегрирование по $d \xi$ ограничено снизу значением $\xi_{\min }=\left(\omega_{\mathrm{pl}} / 2 \varepsilon_{\mathrm{F}}\right)^{2}, \omega_{\mathrm{pl}}$ - энергия плазмона, поскольку не учитывается вклад коллективной моды. Сделаем два дополнительных замечания. Первое: оба коэффициента переноса (8а) и (13a) пропорциональны энергии АП $\bar{\varepsilon}$, что характерно для кинетических коэффициентов в области низких энергий. Второе: функция $f_{2}(\xi)$ в (13б) характеризует вклад корреляционных эффектов в кинетический коэффищиент, вычисленный в приближении Хартри.

\section{4. СОБСТВЕННОЕ ЗНАЧЕНИЕ МАТРИЦЫ РЕЛАКСАЦИИ. КВАЗИТЕМПЕРАТУРА АП}

Используя уравнение (5), стохастическое уравнение для флуктуации внутренней энергии $\delta E_{2}(t)$ можно представить в виде [14]

$$
-\frac{d}{d t} \delta E_{2}(t)+\bar{\varepsilon}\left(\tilde{N} \tilde{\phi} \sigma_{\mathrm{f}}\right) \delta f_{1}\left(F_{21}\right)=F_{21}^{2} L_{\dot{H}_{2} \dot{H}_{2}} \delta\left(\frac{1}{F_{21}}\right)-\zeta(t) .
$$

Для простоты ограничимся учетом двухфононных процессов, поскольку они наиболее эффективны в области высоких температур, представляющих основной интерес. Поэтому в случае АП, движущихся в диэлектрике, кинетический коэффициент в (14) можно заменить на $L_{\dot{H}_{2} \dot{H}_{2}}^{(2)}$. 
Поясним вывод уравнения (14). Во-первых, согласно гипотезе Онзагера флуктуация $\delta E_{2}(t)$ аналогична макроскопическому отклонению физической величины, но отличается от него тем, что флуктуация всегда возникает спонтанно. Поэтому флуктуация $\delta E_{2}(t)$ удовлетворяет уравнению такого же вида, что и уравнение для среднего, но с дополнительным членом $\zeta(t)$ - случайной гауссовской величиной. Во-вторых, состояние газа приближается к квазиравновесию через очень короткое время $(\sim 0,5$ пс). В более грубой временно́й шкале, в шкале переноса тепла, детали образования газа АП, достигшего квазиравновесия, не играют никакой роли. Поэтому из рассмотрения можно исключить эволюцию флуктуаций числа частиц и сохранить только (14). Та же аргументация и то же приближение используются во многих задачах теории неравновесных процессов (каскадных процессов [5], [12], химических реакций [15]).

Согласно неравновесной статистической термодинамике [12], [14] ковариация случайного члена включает величину $\eta=\left(\beta_{2} / \beta_{1}\right)^{2}$ и коэффициент переноса $L_{\dot{H}_{2} \dot{H}_{2}}$. Если же, кроме того, учитывается эффективная масса частиц $\stackrel{*}{m}$ (определение $\stackrel{*}{m}$ дано ниже, см. п. 6.2), то это приводит к появлению множителя $\left(\stackrel{*}{m} / 2 m_{e}\right)^{2}$, как показано в монографии [14]. В результате выражение для ковариации принимает вид

$$
\left\langle\zeta(t) \zeta\left(t^{\prime}\right)\right\rangle=2\left(\frac{\stackrel{*}{m}}{2 m_{e}}\right)^{2} \eta L_{\dot{H}_{2} \dot{H}_{2}} \delta\left(t-t^{\prime}\right)
$$

Согласно флуктуационно-диссипативной теории флуктуации и средние определяются одними и теми же видами взаимодействия. Это общее утверждение согласуется с формулами (14), (15), поскольку ковариация выражается через коэффициент переноса и те самые элементарные процессы рассеяния, которые определяют диссипативный процесс, приводят к флуктуациям энергии подсистемы $\{2\}$.

Воспользовавшись известным соотношением $\delta\left(1 / \beta_{2}\right)=\left(1 / C_{V}\right) \delta E_{2}$, уравнение (14) можно представить в форме релаксационного уравнения

$$
\frac{d}{d t} \delta E_{2}(t)=h \delta E_{2}(t)+\zeta(t)
$$

Величина $h$, вошедшая в релаксационное уравнение, в общем случае является матрицей. Однако в том случае, когда рассматриваются флуктуации только одной переменной (энергии), матрица $h$ вырождается в скаляр, который является единственным собственным значением матрищы релаксации. Учитывая

$$
\frac{\delta f_{1}\left(F_{21}\right)}{\delta\left(1 / F_{21}\right)}=\frac{8}{\pi}\left[\bar{\varepsilon} \varphi\left(F_{21}\right)\right]^{-1}
$$

при $F_{21} \bar{\varepsilon} \gg 1$, сравним $(14)$ с релаксационным уравнением. Из сравнения следует, что

$$
h=\frac{1}{C_{V}}\left\{\frac{8}{\pi} \frac{1}{\varphi\left(F_{21}\right)}\left(\tilde{N} \tilde{\phi} \sigma_{\mathrm{f}}\right)+F_{21}^{2} L_{\dot{H}_{2} \dot{H}_{2}}\right\} .
$$


В стационарном состоянии квазитемпература АП выражается через стационарную корреляционную функцию энергия-энергия (3б). Последняя в свою очередь определяет дисперсию внутренней энергии в стационарном квазиравновесном состоянии

$$
\sigma_{2}^{\mathrm{SS}}=\left(H_{2}, H_{2}\right)_{0}
$$

С учетом вида ковариации (15) запишем флуктуационно-диссипативную теорему. Принимая во внимание, что $h$ является скаляром, имеем [14]

$$
2 \sigma_{2}^{\mathrm{ss}} h=-2\left(\frac{\stackrel{*}{m}}{2 m_{e}}\right)^{2} \eta L_{\dot{H}_{2} \dot{H}_{2}}
$$

Разрешим полученное уравнение относительно дисперсии $\sigma_{2}^{\text {sS }}$. С учетом вида собственного значения матрицы релаксации (16) находим

$$
\sigma_{2}^{\mathrm{sS}}=\frac{\left(\stackrel{*}{m} /\left(2 m_{e}\right)\right)^{2} \eta L_{\dot{H}_{2} \dot{H}_{2}}}{\left(1 / C_{V}\right)\left\{F_{21}^{2} L_{\dot{H}_{2} \dot{H}_{2}}-(8 / \pi)\left(1 / \varphi\left(F_{21}\right)\right)\left(\tilde{N} \tilde{\phi} \sigma_{\mathrm{f}}\right)\right\}} .
$$

Теперь, имея (3б) и (17), нетрудно получить уравнение для $\beta_{2}$ :

$$
\left(\frac{1}{\beta_{2}}\right)^{2}=\frac{\left(\beta_{2} / \beta_{1}\right)^{2}\left(\stackrel{*}{m} /\left(2 m_{e}\right)\right)^{2} L_{\dot{H}_{2} \dot{H}_{2}}}{\left(\beta_{2}\right)^{2} L_{\dot{H}_{2} \dot{H}_{2}}-(8 / \pi)\left(1 / \varphi\left(\beta_{2}\right)\right)\left(\widetilde{N} \tilde{\phi} \sigma_{f}\right)} .
$$

Решение уравнения (18) в случае исчезающе малого потока позитронов $\tilde{\phi} \rightarrow 0$ (нулевое приближение) имеет вид $1 / \beta_{2}=T_{1}\left(\stackrel{*}{m} /\left(2 m_{e}\right)\right)$. Подставляя нулевое приближение в (18), в первом приближении находим

$$
\frac{1}{\beta_{2}}=T_{1} \frac{\stackrel{*}{m} /\left(2 m_{e}\right)}{\sqrt{1-T_{1}^{2}\left(\stackrel{*}{m} /\left(2 m_{e}\right)\right)^{3}\left(\tilde{N} \tilde{\phi} \sigma_{\mathrm{f}}\right) B_{i}}} .
$$

Отметим, что приближенное решение (19) применимо для тех значений $\tilde{\phi}$, которые удовлетворяют условию

$$
\left(\tilde{N} \tilde{\phi} \sigma_{f}\right)<\beta_{1}^{2}\left(\frac{*}{2 m_{e}}\right)^{-3} \frac{1}{B_{i}} .
$$

Величина $B_{i}$ в выражении (19) принимает два значения, которые легко получить, используя (8a) и (13a):

$$
B_{i}=\left\{\begin{array}{lll}
\left(\frac{4}{\pi \bar{\varepsilon}}\right)^{2} \tau_{2} & \text { при } i=2 \text { (двухфононное рассеяние), } \\
\frac{8}{\pi \bar{\varepsilon} \varepsilon_{\mathrm{F}}} \frac{1}{\varphi\left(\beta_{1}\right)} \tau_{3} & \text { при } i=3 \quad \text { (электронное рассеяние). }
\end{array}\right.
$$


Окончательные выражения для времен релаксации из (20а) можно получить, продолжая интегрирование в (8в), (13б):

$$
\begin{gathered}
\frac{1}{\tau_{2}}=\frac{4}{3 \pi^{2}}\left(\frac{g}{M v_{s}^{2}}\right)^{4} \frac{1}{4 \widetilde{N}} \int_{0}^{q_{\mathrm{D}}} d q q^{2} \Delta q \\
\frac{1}{\tau_{3}}=\left(1+\frac{3}{5} \alpha\right)^{-2}\left[\frac{1}{3}\left(1-\xi_{\min }^{3}\right)-\frac{1}{2} \gamma\left(1-\xi_{\min }^{2}\right)+\right. \\
\left.+\gamma^{2}\left(1-\xi_{\min }\right)-\gamma^{3} \ln \left|\frac{1+\gamma}{\xi_{\min }+\gamma}\right|\right]
\end{gathered}
$$

\section{5. ТЕРМАЛИЗАЦИЯ ПОДСИСТЕМЫ АП}

Несмотря на установление квазиравновесия в подсистеме АП с квазитемпературой (20), система в целом остается далекой от полного термодинамического равновесия, поэтому следуюшим этапом эволюции системы является термализация АП. На этом этапе подсистема АП проходит через последовательность состояний текушего квазиравновесия. Эти состояния описываются распределением $\rho_{l}(t)=e^{-S(t, 0)}$, включаюшим термодинамические параметры $F_{i m}(t)$, зависящие от текушего момента времени. Временна́я эволюция параметров $F_{i m}(t)$ описывается системой уравнений [4]

$$
\frac{d}{d t} F_{i m}(t)=\sum_{n j} \frac{\delta^{2} S(t)}{\delta\left\langle P_{m i}\right\rangle_{l}^{t} \delta\left\langle P_{n j}\right\rangle_{l}^{t}} J_{n j}(t),
$$

где $J_{n j}(t)=\left\langle\dot{P}_{n j}\right\rangle_{l}^{t}$ - диссипативные потоки. Чтобы изучить эволюцию квазитемпературы АП, достаточно из системы (21) выделить уравнение с $i=2, m=1$ и сохранить в нем два потока, наиболее существенных с физической точки зрения, а именно

$$
\begin{gathered}
J_{12}=-L_{\dot{H}_{2} \dot{H}_{2}}\left\{\beta_{1}-F_{21}(t)\right\}, \\
J_{22}(t)=n_{\mathrm{V}}\left(N_{2}, N_{2}\right)^{t} k_{\mathrm{V}} .
\end{gathered}
$$

Поток $J_{12}$ определяет отвод энергии из подсистемы $\{2\}$ за счет фононного рассеяния АП и возбуждения электрон-дырочных пар. Поток $J_{22}$ связан с уменьшением энергии подсистемы $\{2\}$ в результате захвата АП тепловыми вакансиями, плотность которых равна $n_{\mathrm{V}}$. В $J_{22}$ также вошла объемная константа скорости захвата АП [1]

$$
k_{\mathrm{V}}= \begin{cases}v(\bar{\varepsilon}) \cdot 2 \pi^{2}\left(\lambda_{\mathrm{B}}^{2} \Gamma(\bar{\varepsilon}) / \widetilde{E}_{\mathrm{b}}\right) & (\text { фононное рассеяние), } \\ v(\bar{\varepsilon}) \cdot 4 \pi\left(\lambda_{\mathrm{B}} / k_{\mathrm{F}}\right) & \text { (электронное рассеяние), }\end{cases}
$$

где $\lambda_{\mathrm{B}}$ - тепловая длина Де Бройля, $Г(\bar{\varepsilon})$ - затухание состояния АП на дне зоны, $\widetilde{E}_{\mathrm{b}}-$ энергия образования комплекса позитрон-вакансия.

Вычислив в рамках стандартной процедуры [4] вторую производную энтропии, с использованием (21), (22) получаем

$$
\frac{d}{d t} \beta_{2}(t)=\left[\beta_{2}(t)\right]^{2}\left\{A(t)\left[\beta_{1}-\beta_{2}(t)\right]+B(t)\right\},
$$


где

$$
\begin{gathered}
A(t)=\frac{1}{c(t)}\left(N_{2}, N_{2}\right)^{t} L_{\dot{H}_{2} \dot{H}_{2}}, \\
B(t)=\frac{1}{c(t)}\left(H_{2}, N_{2}\right)^{t} k_{\mathrm{V}} n_{\mathrm{V}}, \\
c(t)=\left[\beta_{2}(t)\right]^{2}\left\{\left(H_{2}, H_{2}\right)^{t}-\frac{\left(H_{2}, N_{2}\right)^{t}\left(N_{2}, H_{2}\right)^{t}}{\left(N_{2}, N_{2}\right)^{t}}\right\}
\end{gathered}
$$

- неравновесная теплоемкость газа АП, $\beta_{2}(t) \equiv F_{21}(t)$.

Без дополнительных упрошений найти аналитическое решение уравнения (23) невозможно. Выполним необходимые для этого преобразования. Во-первых, будем пренебрегать слабой временно́й зависимостью $A(t), B(t)$ и $c(t)$, записав их в предельном случае $1 / \beta_{2} \rightarrow \infty$. Этот предельный переход, как уже упомянуто выше, соответствует переходу от величин неравновесной термодинамики к величинам механистической статистической теории, в которой числа заполнения состояний постоянны [6], [7]. Во-вторых, введем в выражении (23) новую функцию

$$
u(t)=\frac{1}{\beta_{2}(t)}+a t,
$$

где $a=\beta_{1} A+B$. В результате новая функция будет удовлетворять нелинейному обыкновенному уравнению

$$
\{u(t)-a t\} \frac{d u(t)}{d t}+p=0,
$$

где $p=-A$. Если, кроме того, в полученном уравнении произвести замену $t$ на новую переменную $\chi=-p t$ и соответствуюшую замену функции $u(t)$ на $y(\chi)$, то оно принимает вид уравнения Абеля первого рода:

$$
\left[y(\chi)+\frac{a}{p} \chi\right] \frac{d}{d \chi} y(\chi)=1
$$

Аналитическое решение уравнения (24) может быть представлено только в неявном виде:

$$
\chi=C \exp \left[\frac{a}{p} y(\chi)\right]-\frac{p}{a}\left[y(\chi)+\frac{p}{a}\right],
$$

где $C$ - численный множитель. Известно, что в случае АП в диэлектрике [8] термализация даже при гелиевых температурах практически полностью обусловлена рассеянием АП на тепловых колебаниях атомов решетки. Принимая во внимание это обстоятельство и предполагая, что и в обшем случае захват АП вакансиями вносит достаточно малый вклад в процесс охлаждения подсистемы $\{2\}$, указанньй недостаток решения (25) нетрудно "исправить". А именно, получим (25) в случае исчезающе малой концентрации вакансий $n_{\mathrm{V}} \rightarrow 0$ (нулевое приближение), а затем с использованием нулевого приближения найдем

$$
\frac{1}{\beta_{2}(t)}=\left\{\frac{1}{\beta_{2}}-\frac{2}{3} \beta_{1} L_{\dot{H}_{2} \dot{H}_{2}} t\right\},
$$

где последний член в правой части является поправкой к члену нулевого приближения. При записи (26) было учтено начальное условие: квазитемпература АП в начальный момент термализации, в качестве которого принимается $t=0$, равна $1 / \beta_{2}(19)$. 


\section{6. ЗАКЛЮЧЕНИЕ}

6.1. Затухание и устойчивость состояний АП на дне зоны. Используя известную процедуру вычисления затухания [4], с точностью до величин второго порядка по потенциалу рассеяния АП на фононах получаем

$$
\Gamma_{k}(\omega)=\pi \sum_{\mathbf{q}}\left|V_{1}(q)\right|^{2} \nu\left(\omega_{q}\right)\left\{\delta\left(\omega-\varepsilon_{\mathbf{k}-\mathbf{q}}-\omega_{q}\right)+\delta\left(\omega-\varepsilon_{\mathbf{k}-\mathbf{q}}+\omega_{q}\right)\right\} .
$$

На основе этого выражения можно вычислить затухание состояний АП в диэлектрике на дне зоны образования. В области высоких температур находим

$$
\Gamma(\bar{\varepsilon})=\frac{1}{2 \pi} g^{2} \frac{T_{1}}{M v_{s}^{2} \widetilde{N}}\left(2 m_{e}\right)^{3 / 2}(2 \bar{\varepsilon})^{1 / 2} .
$$

Оценка затухания $(27)$ в случае АП в диэлектрике приводит к результату $\Gamma(\bar{\varepsilon}) \ll \widetilde{E}_{\mathrm{b}}$. Другая оценка была получена в случае АП в шелочных металлах: $\Gamma(\bar{\varepsilon}) \simeq \widetilde{E}_{\mathrm{b}}$. Отсюда следует, что в диэлектриках состояние АП на дне зоны хорошо определено, тогда как в металлах столь же хорошо определенное состояние АП отсутствует. В металлах эта ситуация возникает из-за быстрых диссипативных процессов, обусловленных возбуждением пар вблизи поверхности Ферми.

Согласно критерию неравновесной статистической термодинамики [14] стационарное состояние, далекое от равновесия, является термодинамически устойчивым, если все собственные значения матрицы релаксации имеют отрицательные вещественные части. В рассмотренном нами случае имеется только одно собственное значение (16), и условие устойчивости принимает очень простой вид: $h<0$. Последнее ограничивает область допустимых значений квазитемпературы АП (19) при заданной скорости образования АП:

$$
\left(\frac{1}{\beta_{2}}\right)<\left[\frac{\pi^{2}}{16} \bar{\varepsilon} L_{\dot{H}_{2} \dot{H}_{2}} \frac{1}{\widetilde{N} \tilde{\phi} \sigma_{f}}\right]^{1 / 3} .
$$

6.2. Квазитемпература АП в неравновесном стационарном состоянии. Выражение (19) представляет квазитемпературу АП $\left(1 / F_{21}\right)_{\mathrm{ss}} \equiv 1 / \beta_{2}$ как перенормированную температуру термостата $T_{1}$, причем согласно (19) перенормировка связана с учетом трех факторов. Первый определяется скоростью формирования газа АП в твердом теле $\tilde{N} \tilde{\phi} \sigma_{\mathrm{f}}$. Второй фактор обусловлен кинетическими эффектами взаимодействия. Чтобы представить его в явном виде, запишем действительную часть массового оператора, соответствуюшего состоянию АП на дне зоны:

$$
\operatorname{Re} \widetilde{M}(\bar{\varepsilon})=\frac{4 m_{e} T_{1}}{\pi^{2} v_{s} q_{\mathrm{D}}}\left|V_{1}\left(q_{\mathrm{D}}\right)\right|^{2}\left\{\frac{4 m_{e}}{q_{\mathrm{D}}^{2}}\left(\bar{\varepsilon}+\frac{k^{2}}{4 m_{e}}\right)-1\right\}=\varkappa_{1} \bar{\varepsilon}+\varkappa_{2} \frac{k^{2}}{4 m_{e}}+\Delta \mu_{2} .
$$

Здесь $\Delta \mu_{2}-$ сдвиг химического потенциала подсистемы $\{2\}, k$-импульс АП. Поскольку эффективная масса выражается через $\varkappa_{1}$ и $\varkappa_{2}$ как

$$
\frac{*}{2 m_{e}}=\frac{1-\varkappa_{1}}{1+\varkappa_{2}}
$$




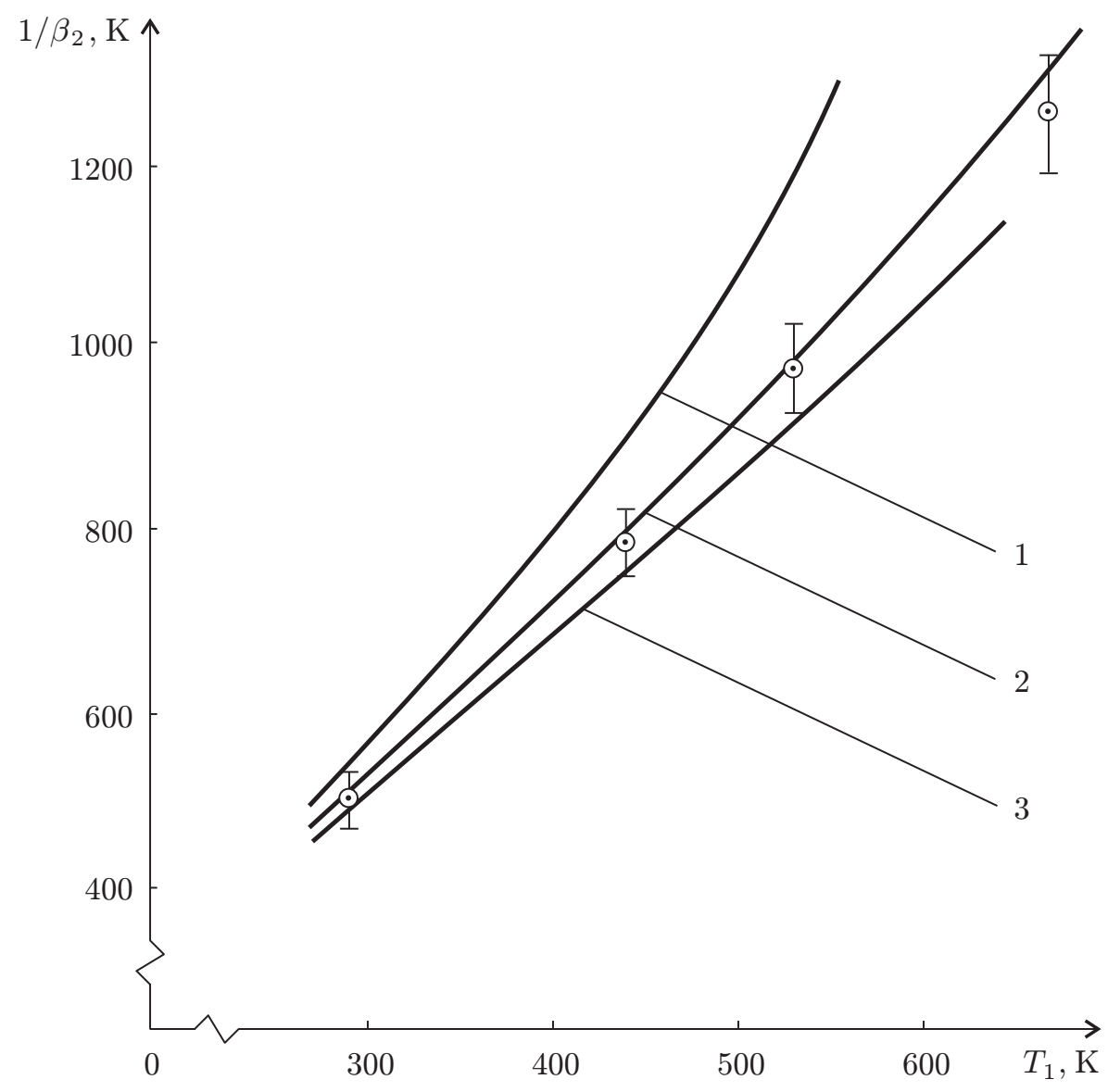

то вклад эффектов взаимодействия в (19) определяется множителями $\stackrel{*}{m} /\left(2 m_{e}\right)$. Наконец, третий перенормируюший фактор обусловлен динамическими эффектами столкновений и описывается с помошью времен релаксации $\tau_{2}$ и $\tau_{3}(20 б),(20$ в), вошедших в $B_{i}(20 \mathrm{a})$.

Формула (19) дает семейство кривых, некоторые из которых представлены на рисунке (кривая 1 соответствует значению $\left(\tilde{N} \tilde{\phi} \sigma_{\mathrm{f}}\right) B_{2}=65$ эВ ${ }^{-2}$, кривая $2-$ значению 27 э $\mathrm{B}^{-2}$, кривая 3 - значению 8 э $\mathrm{B}^{-2}$ ). Как показано на рисунке, теоретическая кривая 2 удовлетворительно согласуется с данными эксперимента, полученными на основе анализа узкой компоненты распределения АП по импульсам в кварце [8].

Сделаем еще два замечания. Во-первых, как следует из рисунка, квазитемпература АП все заметнее отклоняется от линейной функции по мере роста температуры термостата. Во-вторых, если при постоянном значении температуры $T_{1}=$ const увеличивать поток позитронов $\tilde{\phi}$, оставаясь при этом в пределах применимости формулы (19), то квазитемпература АП (см. рисунок) также растет.

6.3. Два подхода в теории термализации. Время термализации АП. Возможны два направления исследования термализации быстрых частиц в твердых телах: 
феноменологический подход [16], [17], основанный на уравнении теплопроводности, и предложенный нами подход, в котором система описывается на термодинамическом уровне.

Трудность феноменологической теории заключается не столько в отыскании решения уравнения теплопроводности, сколько в применимости самой концепции теории теплопроводности. Дело в том, что в качестве источника теплового пика в работах [16], [17] рассматриваются "горячие" атомы (АП, каскадные частицы, позитроны и т.п.). При этом энергия, которую они передают решетке, высвобождается в небольшом объеме среды. Можно ли в таком случае считать, что линейное уравнение теплопроводности, описывающее распространение тепла в непрерывной среде, применимо? Кроме того, изучение процесса охлаждения в условиях большой разницы температур двух подсистем неминуемо должно привести к квазилинейному уравнению теплопроводности, а не к линейному [16].

Стандартное решение уравнения теплопроводности включает два равноценных множителя: множитель, спадающий с ростом времени, и множитель, который со временем растет. По-видимому, то, что авторы работ [16], [17] полностью отбрасьвают возрастающий множитель, продиктовано стремлением достигнуть заметного спада температуры. В этом случае временна́я эволюция температуры “горячих" атомов определяется функцией $\sim t^{-3 / 2}$, которая имеет сингулярность при $t=0$. Чтобы избежать указанной трудности, предлагается [17] либо ввести стартовое время $t_{0}$, используя в качестве решения функцию $\sim\left(t+t_{0}\right)^{-3 / 2}$, либо ограничить во всех интегральных преобразованиях нижний предел интегрирования. Конечно, все это вносит в теорию дополнительную неясность.

Представленная здесь термодинамическая теория термализации не подтверждает отправную точку рассуждений авторов работ [16], [17]. Во-первых, на основе кинетических уравнений (21) установлено, что квазитемпература АП удовлетворяет уравнению, которое преобразуется в уравнение Абеля первого рода (24). Естественно, оно не имеет сходства с линейным уравнением параболического типа, использованным в работе [16]. Во-вторых, найдено точное решение (25) полученного нами уравнения. На основе (25) в условиях, когда захват АП вакансиями дает малый вклад в процесс охлаждения, показано, что временно́й ход квазитемпературы (26) близок к линейному спаду. Отклонение от линейности согласно (26) описывается слабо меняющейся логарифмической функцией.

Найдем аналитическое выражение для $\tau_{\mathrm{T}}$ - времени термализации АП в диэлектриках. Для этого достаточно вычислить квазитемпературу АП в момент окончания процесса термализации. Если в (26) подставить $L_{\dot{H}_{2} \dot{H}_{2}}^{(2)}$ в виде $(8 \mathrm{a})$ и пренебречь поправочным (логарифмическим) членом, то после замены $t$ и $\bar{\varepsilon}$ на $\tau_{\mathrm{T}}$ и $\bar{\varepsilon}_{\mathrm{T}}$ получим

$$
\frac{1}{\beta_{2}\left(\tau_{\mathrm{T}}\right)}=\frac{1}{\beta_{2}}-\frac{2}{3} \bar{\varepsilon}_{\mathrm{T}} \tau_{\mathrm{T}} \frac{1}{\tau_{2}} .
$$

Здесь $\bar{\varepsilon}_{\text {T }}$ - средняя энергия АП в случае тепловых скоростей. Теперь, приравнивая $1 / \beta_{2}\left(\tau_{\mathrm{T}}\right)$ к температуре термостата $T_{1}$, находим из полученного таким образом урав- 
нения искомое выражение

$$
\tau_{\mathrm{T}}=\frac{3}{2}\left(\frac{1}{\beta_{2}}-T_{1}\right) \frac{\tau_{2}}{\bar{\varepsilon}_{\mathrm{T}}}
$$

В отличие от результата, полученного в работе [11], время $\tau_{\mathrm{T}}(28)$ не зависит от температуры термостата, поскольку $1 / \beta_{2} \sim T_{1}$ (19) и $\bar{\varepsilon}_{\mathrm{T}} \sim T_{1}$. Оценка $\tau_{\mathrm{T}}(28)$ показала, что время термализации равно нескольким пикосекундам и много меньше времени аннигилящии АП [1], [8].

\section{Список литературы}

[1] W. Brandt. Appl. Phys. 1974. V. 5. № 1. P. 1.

[2] C. H. Hodges, M. J. Stott. Phys. Rev. B. 1973. V. 7. № 1. P. 73.

[3] N. D. Lang, W. Kohn. Phys. Rev. B. 1971. V. 3. № 4. P. 1215.

[4] Д. Н. Зубарев. Неравновесная статистическая термодинамика. М.: Наука, 1977.

[5] Ю. А. Кашлев. ТМФ. 2000. Т. 123. № 3. С. 485.

[6] Ю. А. Кашлев. ТМФ. 1995. Т. 102. № 1. С. 106.

[7] Д. Карери. Порядок и беспорядок в структуре материи. М.: Мир, 1985.

[8] H. Ikari. J. Phys. Soc. Japan. 1979. V. 46. № 1. P. 97.

[9] W. Brandt, R. Paulin. Phys. Rev. Lett. 1973. V. 31. № 19. P. 1214.

[10] Д. Пайнс. Элементарные возбуждения в твердых телах. М.: Мир, 1965.

[11] J. P. Carbotte, H. L. Arora. Can. J. Phys. 1967. V. 45. P. 387.

[12] Ю. А. Кашлев. ТМФ. 2001. Т. 126. № 2. С. 311.

[13] Л. Каданов, Г. Бейм. Квантовая статистическая механика. Методы функций Грина в теории равновесных и неравновесных процессов. М.: Мир, 1964.

[14] Дж. Кайзер. Статистическая термодинамика неравновесных процессов. М.: Мир, 1990.

[15] E. C. Zimmerman, J. Ross. J. Chem. Phys. 1984. V. 80. P. 720.

[16] F. Seits, J. S. Kochler. Solid State Phys. 1956. V. 2. P. 305.

[17] R. Kelly. Rad. Eff. 1977. V. 32. P. 91.

Поступила в редакцию 22.XII.2003 г., после доработки 9.III.2004 г. 